\title{
Blue Straggler Stars in Galactic Globular Clusters: Tracing the Effect of Dynamics on Stellar Evolution
}

\author{
Francesco R. Ferraro ${ }^{1}$ and Barbara Lanzoni ${ }^{1}$ \\ ${ }^{1}$ Dipartimento di Astronomia, Università di Bologna, \\ Via Ranzani 1, 40127 Bologna, Italy \\ email: francesco.ferraro3@unibo.it - barbara.lanzoni@bo.astro.it
}

\begin{abstract}
In this contribution we review the main observational properties of Blue Stragglers Stars (BSS) in galactic GCs. A flower of results on the BSS frequency, radial distribution, and chemical composition are presented and discussed.
\end{abstract}

Keywords: globular clusters: general; stars: evolution; binaries: general; blue stragglers

\section{Introduction}

Ultra-dense cores of Galactic Globular Clusters (GCs) are very efficient "furnaces" for generating exotic objects, such as low-mass X-ray binaries, cataclysmic variables, millisecond pulsars, blue stragglers (BSS), etc. Most of these stars are thought to be the by-products of the evolution of binary systems, possibly originated and/or hardened by stellar interactions. Thus, studying the nature of these exotic objects and the properties of artificial sequences, as that of BSS, in the color-magnitude diagrams (CMDs) of GCs can serve as a powerful diagnostic of the dynamical evolution of clusters, and of its effects on the evolution of their stellar population and binary systems (see Bailyn 1995 and reference therein).

This topic has received strong impulse in the recent years. In this paper we review the main properties of the most known exotic population of GCs: the so-called BSS, that describe the very first sequence of exotic objects discovered in the CMD.

First discovered by Sandage (1953) in M3, BSS are commonly defined as stars brighter and bluer (hotter) than the main sequence (MS) turnoff along an apparent extension of the MS. Thus, they mimic a rejuvenated stellar population and their existence has been a puzzle for many years. Direct measurements (Shara et al. 1997) and indirect evidences show that BSS are more massive than the normal MS stars, pointing toward stellar mergers as possible explanation for their origin. Indeed, their formation mechanisms are not yet completely understood, and the leading explanations, at present, involve mass transfer (MT) between binary companions (McCrea 1964; Zinn \& Searle 1976), possibly up to the complete coalescence of the binary system, or the merger of stars (whether or not in binaries) induced by collisions (COL; Hills \& Day 1976). Thus, BSS represent the link between standard stellar evolution and the effects of cluster dynamics (see Bailyn 1995).

\section{The UV approach to the study of BSS}

The observational and interpretative scenario of BSS has significantly changed in the last 20 years. In fact, for almost 40 years since their discovery, BSS have been detected only in the outer regions of GCs or in relatively loose clusters, thus generating the idea 


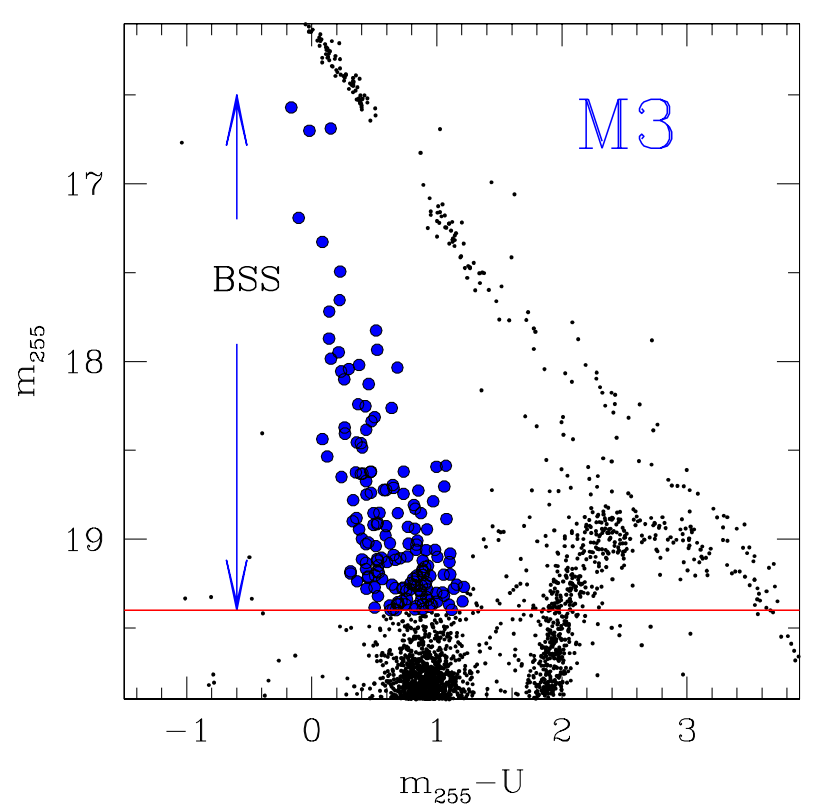

Figure 1. BSS in the UV: the case of M3. The horizontal line at $m_{255}=19.4$ is the assumed limiting magnitude, corresponding to $\sim 5 \sigma$ above the turnoff level (from F97).

that low-density environments were their natural habitats. However, this was just an observational bias, and, starting from the early '90, high resolution studies allowed to properly image and discover BSS also in the highly-crowded central regions of dense GCs (see the case of NGC 6397 by Auriere et al. 1990). In particular, the advent of the Hubble Space Telescope (HST) represented a really turning point in BSS studies, thanks to its unprecedented spatial resolution and imaging/spectroscopic capabilities in the ultraviolet (UV; see Paresce et al. 1991, Ferraro \& Paresce 1993, Guhathakurta et al. 1994, etc).

In fact, the systematic study of BSS, especially in the central regions of high density clusters, still remains problematic in the optical bands, even if using HST. This is because the CMD of old stellar populations in the classical $(V, B-V)$ plane is dominated by the cool stellar component. Hence, the observation and the construction of complete samples of hot stars (as BSS, other by-products of binary system evolution, extreme blue horizontal branch stars, etc.) is "intrinsically" difficult in this plane. Moreover, BSS can be easily mimicked by photometric blends of red giant (RGB) stars in the optical CMDs. Instead, at UV wavelengths RGB stars are very faint, while BSS are among the brightest objects. In particular, BSS define a narrow, nearly vertical sequence spanning $\sim 3 \mathrm{mag}$ in the UV plane (see Fig. 1), thus being much more easily recognizable, while BSS-like blends are much less severe at these wavelengths because of the relative faintness of sub-giant and RGB stars. Indeed, the $\left(m_{255}, m_{255}-m_{336}\right)$ plane is an ideal tool for selecting BSS even in the densest cores of GCs, and its systematic use allowed to put the BSS study into a more quantitative basis than ever before (e.g., Ferraro et al. 2003; see Fig. 2). 


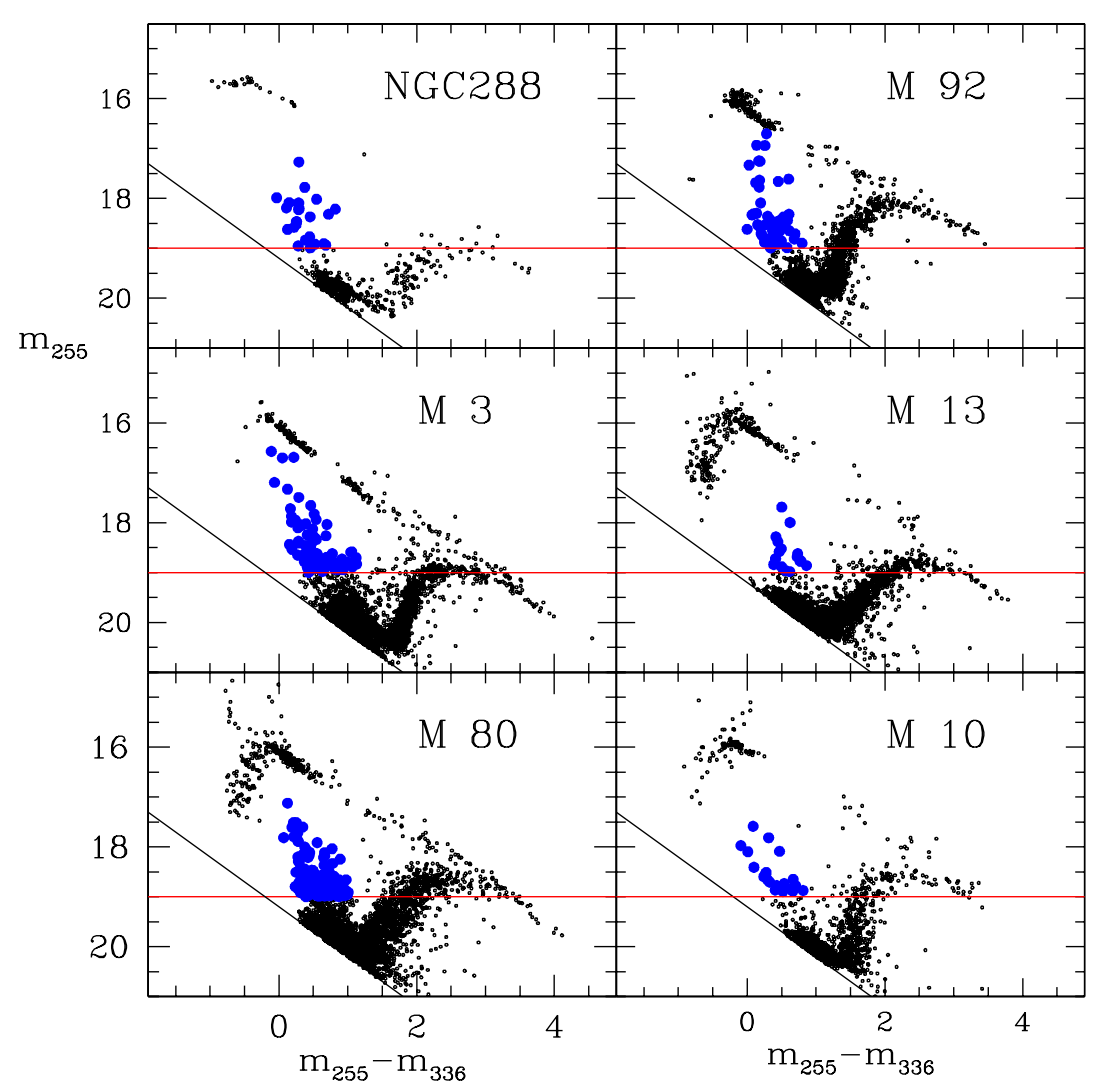

Figure 2. $\left(m_{255}, m_{255}-m_{336}\right)$ CMDs for the six clusters discussed in F03. Horizontal and vertical shifts have been applied to all CMDs in order to match the main sequences of M3. The horizontal solid line corresponds to $m_{255}=19$ in M3. The bright BSS candidates are marked as large filled circles.

\section{BSS specific frequency}

Based on these observations, the first catalogs of BSS have been published (e.g., Fusi Pecci et al. 1992; Ferraro, Fusi Pecci \& Bellazzini 1995, hereafter FFB95), until the most recent collection of BSS which counts nearly 3000 candidates i 56 Galactic GCs (Piotto et al. 2004; the most recent results based on this data-set are discussed by Leigh et al. in this volume).

These works have significantly contributed to form the nowadays commonly accepted idea that BSS are a normal stellar population in GCs, since they are present in all properly observed clusters. However, according to Fusi Pecci et al. (1992), BSS in different environments could have different origins. In particular, BSS in loose GCs might be produced by the coalescence of primordial binaries, while in high density GCs (depending on survival-destruction rates for primordial binaries) BSS might arise mostly from stellar interactions, particularly those which involve binaries. While the suggested mechanisms of BSS formation could be at work in clusters with different densities (FFB95; Ferraro et al. 1999), there are evidences that they could also act simultaneously within the same 


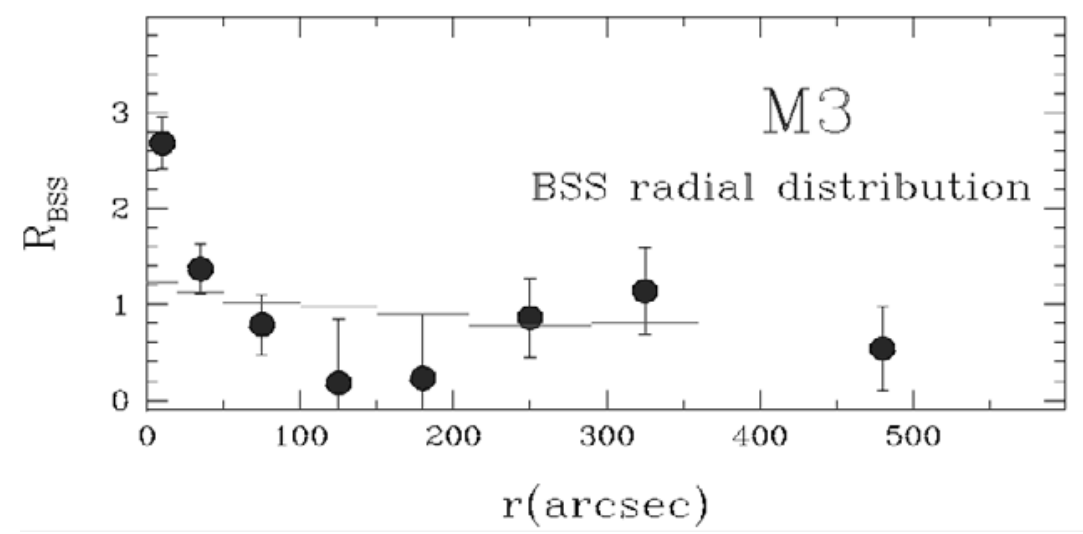

Figure 3. The relative frequency of BSS in M3 is plotted as a function of the radial distance from the cluster center. The horizontal lines show the relative frequency of the RGB stars used as a comparison population. For $r>6^{\prime}$ only the relative frequency of BSS has been computed using the Sandage (1953) candidates. (From F97)

cluster (as in the case of M3; see Ferraro et al. 1993, hereafter F93; Ferraro et al. 1997, hereafter F97).

A number of interesting results have been obtained from cluster-to-cluster comparisons. For this purpose we used the BSS specific frequency, defined as the number of BSS counted in a given region of the cluster, normalized to the number of "normal" cluster star in the same region, adopted as reference (generally we adopted the horizontal branch stars, hereafter HB). The BSS specific frequency has been found to largely vary from cluster to cluster: for the six GCs considered by Ferraro et al. (2003), the BSS frequency varies from 0.07 to 0.92 , and does not seem to be correlated with central density, total mass, velocity dispersion, or any other obvious cluster property (see also Piotto et al. 2004). Even "twin" clusters as M3 and M13 harbor a quite different BSS populations: the specific frequency in M13 is the lowest ever measured in a GC (0.07), and it turns out to be 4 times lower than that measured in M3 (0.28). Which is the origin of this difference? The paucity of BSS in M13 suggests either that the primordial population of binaries in M13 was poor, or that most of them were destroyed. Alternatively, as suggested by F97, the mechanism producing BSS in the central region of M3 is more efficient than in M13, because the two systems are in different dynamical evolutionary phases.

In this respect, the most surprising result is that the largest BSS specific frequency has been found in two GCs which are at the extremes of central density values in our sample: NGC 288 and M80, with the lowest and the highest central density, respectively. This suggests that the two formation channels can have comparable efficiency in producing BSS in their respective typical environment.

\section{The BSS radial distribution}

M3 has played a fundamental role in the BSS history, because it is the GC not only where BSS have been first identified, but also where the BSS radial distribution has been studied for the first time over the entire cluster extension. In fact by combining UV HST observations of the cluster central region (F97) and extensive wide field ground-based observations (F93; Buonanno et al. 1994), F97 presented the BSS radial distribution 


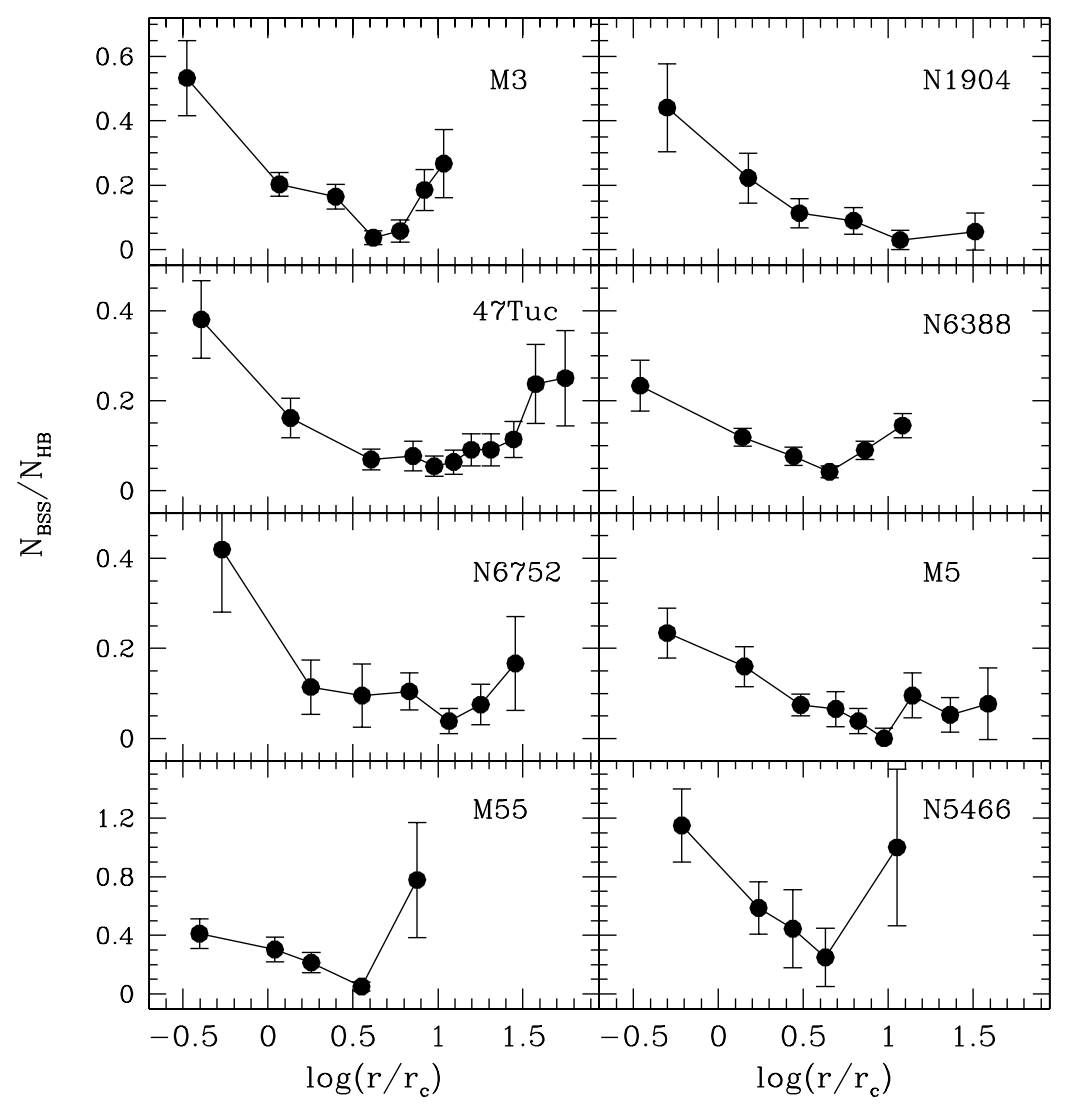

Figure 4. The BSS radial distribution observed in 8 GCs. In all cases, but NGC 1904, it is clearly bimodal, with a peak in the center, a dip at intermediate radii, and an upturn in the external regions.

of M3 all over its radial extent $\left(r \sim 6^{\prime}\right)$. The resulting distribution was completely unexpected: BSS appear to be more centrally concentrated than RGB stars in the central regions, and less concentrated in the cluster outskirts.

For further investigating such a surprising result, F97 divided the surveyed area in a number of concentric annuli, and counted the number of BSS and RGB stars normalized to the sampled luminosity in each annulus, accordingly to the following relations (F93):

$$
R_{\mathrm{BSS}}=\frac{\left(N_{\mathrm{BSS}} / N_{\mathrm{BSS}}^{\mathrm{tot}}\right)}{\left(L^{\text {sample }} / L_{\text {tot }}^{\text {sample }}\right)}
$$

and

$$
R_{\mathrm{RGB}}=\frac{\left(N_{\mathrm{RGB}} / N_{\mathrm{RGB}}^{\mathrm{tot}}\right)}{\left(L^{\text {sample }} / L_{\mathrm{tot}}^{\mathrm{sample}}\right)},
$$

respectively. The result is shown in Fig. 3, and clearly shows that the radial distribution of BSS in M3 is bimodal: it reaches maximum at the center of the cluster, shows a clearcut dip in the intermediate region (at $100^{\prime \prime}<r<200^{\prime \prime}$ ), and rises again in the outer region (out to $r \sim 360^{\prime \prime}$ ). 


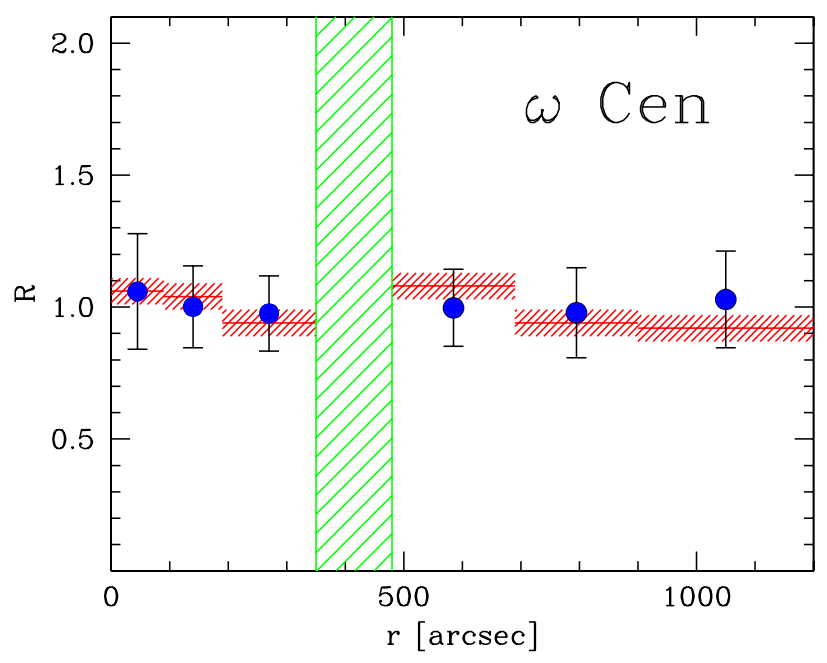

Figure 5. The double-normalized relative frequency $R_{B S S}$ of the BSS (filled circles) in $\omega$ Cen. The shaded area marks the cluster region we excluded in order to avoid incompleteness problems. The horizontal lines show the relative frequency of the RGB stars used as reference population. (From Ferraro et al. 2006a).

While the bimodality detected in M3 was considered for years to be peculiar, the most recent results demonstrated that this is not the case. In fact, in the last years the same observational strategy adopted by F97 in M3 has been applied to a number of other clusters, with the aim of studying the BSS radial distribution over the entire cluster extension. Bimodal distributions with an external upturn have been detected in several cases (see Fig. 4): 47 Tuc (Ferraro et al. 2004), NGC 6752 (Sabbi et al. 2004), M55 (Zaggia et al. 1997; Lanzoni et al. 2007c), M5 (Warren et al. 2006; Lanzoni et al. 2007a), NGC 6388 (Dalessandro et al. 2007), NGC 5466 (Beccari et al. 2007, in preparation).

Originally, F97 argued that the bimodal distribution of BSS in M3 might be the signature of the two formation mechanisms acting simultaneously in the same cluster: the external BSS would arise from MT activity in primordial binaries, while the central BSS would be generated by stellar collisions leading to mergers. Sigurdsson et al. (1994) offered another explanation for the bimodal BSS distribution in M3. They suggested that all BSS were formed in the core and then ejected to the outer regions by the recoil from the interactions. Those BSS which get kicked out to a few core radii would rapidly drift back to the center of the cluster due to mass segregation, thus leading to the central BSS concentration and a paucity of BSS in the intermediate regions (around a few core radii). More energetic recoils would kick the BSS to larger distances and, since stars require much more time to drift back toward the core, these may account for the overabundance of BSS in the cluster outskirts. We are currently using Monte-Carlo dynamical simulations in order to discern between different possibilities (see also the contribution by Lanzoni in this book). Mapelli et al. (2004, 2006) and Lanzoni et al. (2007a) modeled the dynamical evolution of BSS in a number of clusters, by using a modified version 


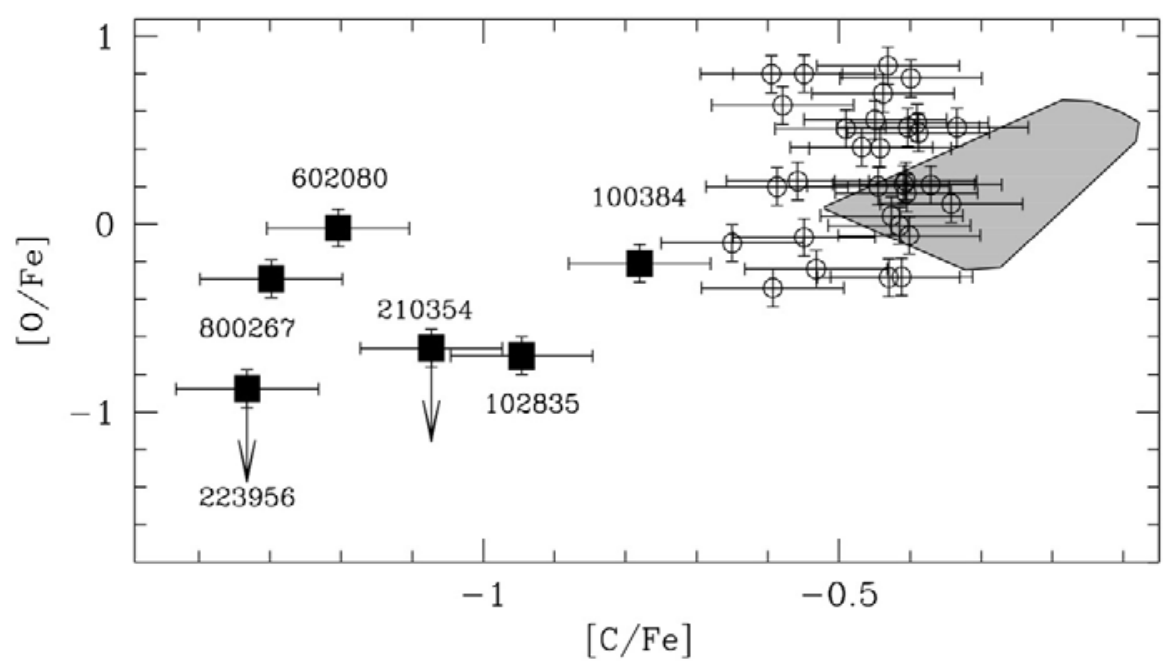

Figure 6. $[\mathrm{O} / \mathrm{Fe}]$ ratio as a function of $[\mathrm{C} / \mathrm{Fe}]$ for the BSS observed in 47 Tuc. Normal BSS are marked with empty circles, while CO-depleted BSS are marked with filled squares and their names are also reported. The gray regions correspond to the location of the 12 turnoff stars in 47 Tuc analyzed by Carretta et al. (2005).

of the code described by Sigurdsson \& Phinney (1995). Their results demonstrate that the observed BSS bimodal distributions cannot be explained within a purely collisional scenario in which all BSS are generated in the core through stellar interactions. In fact, an accurate reproduction of the external upturn of the BSS radial distribution can be obtained only by requiring that a sizable $(\sim 20-40 \%)$ fraction of BSS is generated in the peripheral regions, where primordial binaries can evolve in isolation and experience mass transfer processes without suffering significant interactions with other cluster stars.

Even if the number of the surveyed clusters is low, the bimodal radial distribution first found in M3 and thought to be peculiar could instead be the natural one. However, generalizations cannot be made from a sample of a few clusters only, and such a statement needs to be characterized on a much more solid statistical base. Indeed, two exceptions are already known: NGC 1904, which does not present any external upturn (Lanzoni et al. 2007b), and $\omega$ Cen (Ferraro et al. 2006a), which shows a completely flat BSS radial distribution.

The case of $\omega$ Cen deserves specific comments: by using a proper combination of HST high-resolution data and wide-field ground-based observations sampling the entire radial extension of the cluster, Ferraro et al. (2006a) have detected the largest population of BSS ever observed in any stellar system: more than 300 candidates have been identified. At odds with all the GCs previously surveyed, BSS in $\omega$ Cen have been found not to be centrally segregated with respect to the other cluster stars (see Fig. 5). This is the cleanest evidence ever found that $\omega$ Cen is not fully relaxed, even in the central regions, and it suggests that the observed BSS are the progeny of primordial binaries, whose radial distribution was not yet significantly altered by stellar collisions and by the dynamical evolution of the cluster. Hence, most of these objects should have been produced essentially by MT processes, and the population of BSS in $\omega$ Cen could represent the purest and largest population of non-collisional BSS ever observed. Thus, $\omega$ Cen represents the best laboratory for studying the physical and chemical properties of MTBSS. 


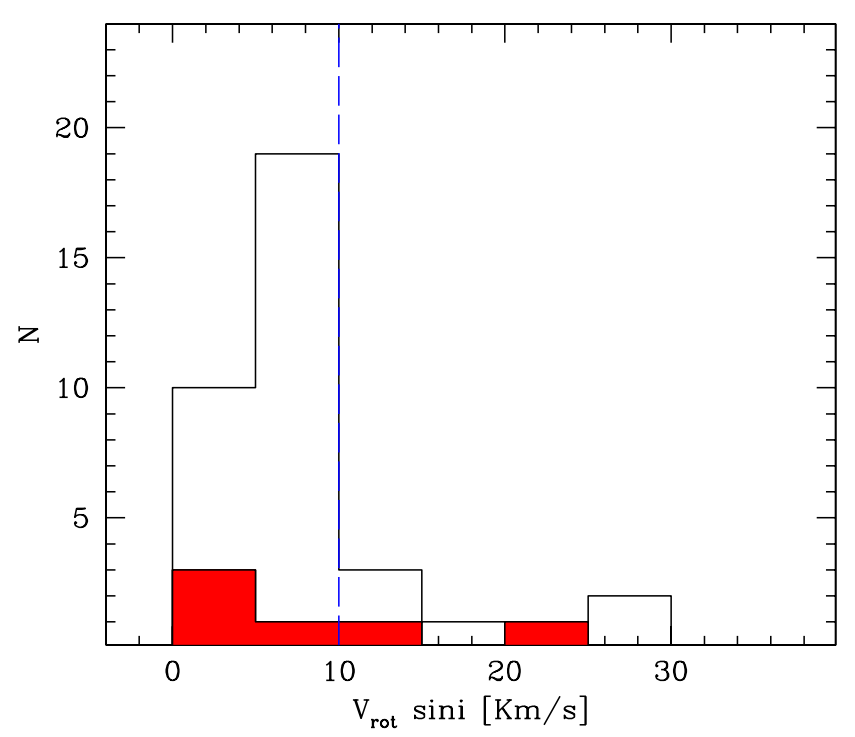

Figure 7. The rotation velocity distribution of normal BSS (empty histogram) is compared to the CO-depleted BSS distribution (filled histogram). The vertical dashed line marks the separation between slow and the 10 "fast" rotators (with $V_{\text {rot }} \sin i>10 \mathrm{~km} / \mathrm{s}$ ).

\section{Searching for the chemical signature of the BSS formation process}

Theoretical models still predict conflicting results on the expected properties of BSS generated by different production channels. For instance, Benz \& Hills (1987) predict high rotational velocities for COL-BSS, whereas Leonard \& Livio (1995) have shown that a substantial magnetic braking could occur, and the resulting BSS are not fast rotators. In the case of BSS formed through the MT production channel, rotational velocities larger than those of typical MS stars are predicted (Sarna \& de Greve 1996). Concerning the chemical surface abundances, hydrodynamic simulations (Lombardi et al. 1995) have shown that very little mixing is expected to occur between the inner cores and the outer envelopes of the colliding stars. On the other hand, signatures of mixing with incomplete CN-burning products are expected at the surface of BSS formed via the MT channel, since the gas at the BSS surface is expected to come from deep regions of the donor star, where the CNO burning was occurring (Sarna \& de Greve 1996).

Spectroscopic observations have recently begun to provide the first set of basic properties of BSS (effective temperature, mass, rotation velocity, etc.; see the recent work by De Marco et al. 2005). However, with the exception of a few bright BSS in the open cluster M67 (Mathys 1991; Shetrone \& Sandquist 2000), an extensive survey of BSS surface abundance patterns is still lacking, particularly in GCs. In this context the advent of 8-meter class telescopes equipped with multiplexing capability spectrographs is giving a new impulse to the study of the BSS properties. By using FLAMES at the ESO VLT we are currently performing extensive surveys of surface abundance patterns for representative numbers of BSS in a sample of Galactic GCs. The first results of this search have lead to an exciting discovery: by measuring the surface abundance patterns of 43 BSS in 47 Tuc, we discovered a sub-population of BSS with a significant depletion of 


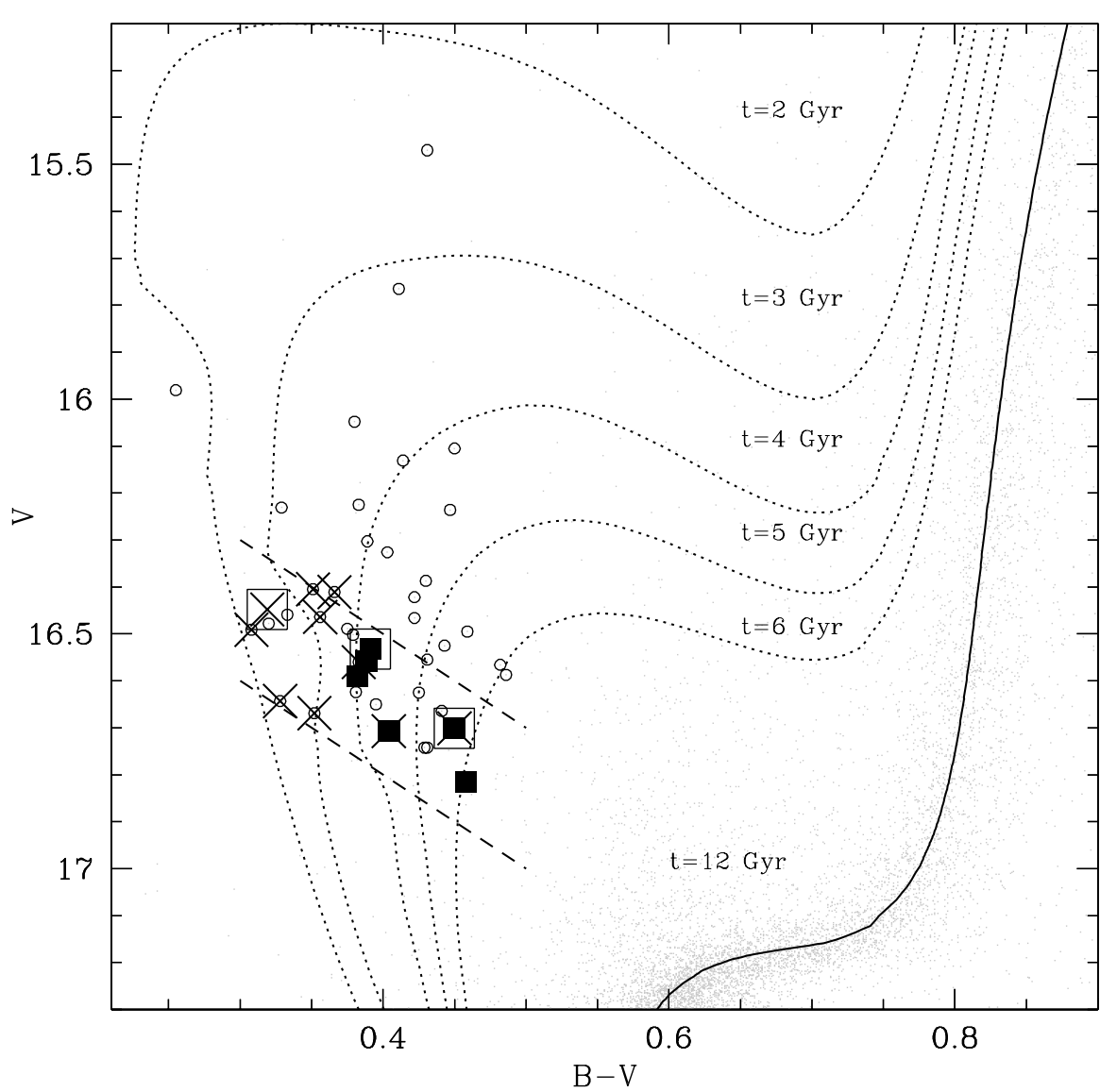

Figure 8. Zoomed CMD of 47 Tuc in the BSS region. Normal BSS are marked with open circles, while CO-depleted BSS are shown as filled squares. Isochrones of different ages (from 2 to 12 Gyr) from Cariulo et al. (2003) are overplotted for comparison. The three W UMa systems and the 10 BSS rotating with $V_{\text {rot }} \sin i>10 \mathrm{~km} / \mathrm{s}$ are highlighted with large empty squares and large crosses, respectively.

Carbon $(\mathrm{C})$ and Oxygen $(\mathrm{O})$, with respect to the dominant population (see Fig. 6). This evidence is interpreted as the presence of CNO burning products on the BSS surface, coming from a deeply peeled parent star, as expected in the case of the MT formation channel. Thus, our discovery in 47 Tuc could be the first detection of a chemical signature clearly pointing to the MT formation process for BSS in a GC.

Indeed, the acquired data-set is a gold-mine of informations. In fact, our observations have shown that (1) only 10 BSS have been found to show rotational velocities larger than $v \sin i>10 \mathrm{Km} / \mathrm{s}$, while most of the BSS are slow rotators at odds with what canonical models predict (see Fig. 7); (2) BSS with CO depletions and the few BSS with $v \sin i>10 \mathrm{Km} / \mathrm{s}$ appear "less evolved" than the others: they all lie within a narrow strip at the faint-end of the BSS luminosity distribution in the CMD (see Fig. 8); (3) some of them are WUma binary systems, suggesting that the evolution of these systems could be a viable channel for the formation of BSS in GCs. 


\section{References}

Auriere, M., Lauzeral, C., \& Ortolani, S. 1990, Nature, 344, 638

Bailyn, C. D. 1995, ARA $8 A, 33,133$

Bellazzini, M., et al. 2002, AJ, 123, 1509

Benz, W. \& Hills, J. G. 1987, ApJ, 323, 614

Buonanno, R., et al. 1994, A\&A A, 290, 69

Cariulo, P., Degl'Innocenti, S., \& Castellani, V., 2003, A\&A, 412, 1121

Carretta, E., Gratton, R. G., Lucatello, S., Bragaglia, A., \& Bonifacio, P. 2005, A\&A, 433, 597

Dalessandro, E., et al. 2007, ApJ, submitted

De Marco, O., et al. 2005, ApJ,632, 894

Ferraro, F. R. \& Paresce, F., 1993, AJ, 106,154

Ferraro F. R., et al. 1993, AJ, 106, 2324 (F93)

Ferraro, F. R., Fusi Pecci, F., \& Bellazzini, M. 1995, A\&A A, 294, 80 (FFB95)

Ferraro, F. R., et al. 1997, A\& A, 324, 915 (F97)

Ferraro, F. R., Paltrinieri, B., Rood, R. T., \& Dorman, B. 1999a, ApJ, 522, 983

Ferraro, F. R., et al. 2003, ApJ, 588, 464

Ferraro, F. R., et al. 2004, ApJ, 603, 127

Ferraro, F. R., et al. 2006a, ApJ, 638, 433

Ferraro, F. R., et al. 2006b, ApJ 647, L53

Fusi Pecci, F., et al. 1992, AJ, 104, 1831

Guhathakurta, P., et al. 1994, AJ, 108, 1786

Hills, J. G., \& Day, C. A. 1976, Astrophys. Lett., 17, 87

Lanzoni, B., et al. 2007a, ApJ, 663, 267

Lanzoni, B., et al. 2007b, ApJ 663, 1040

Lanzoni, B., et al. 2007c, $A p J$ in press

Leonard, P. J. T. \& Livio, M. 1995, ApJ, 447L, 121

Lombardi, J. C. Jr., Rasio, F. A., \& Shapiro, S. L. 1995, ApJ, 445, L117

Mapelli, M., et al. 2004, ApJ, 605, L29

Mapelli, M., et al. 2006, MNRAS, 373, 361

Mathys, G. 1991, A\& $A, 245,467$

McCrea, W. H. 1964, MNRAS, 128, 147

Paresce, F., et al. 1991, Nature, 352, 297

Piotto, G., et al. 2004, ApJ, 604, L109

Sabbi, E., Ferraro, F. R., Sills, A., \& Rood, R. T. 2004, ApJ, 617, 1296

Sandage A. R. 1953, AJ, 58, 61

Sarna, M. J. \& de Greve, J. P. 1996, QJRAS, 37, 11

Shara, M. M., Saffer, R. A., \& Livio, M. 1997, ApJ, 489, L59

Shetrone, M. D. \& Sandquist, E. L. 2000, AJ, 120, 1913

Sigurdsson, S., Davies, M. B., \& Bolte, M. 1994, ApJ, 431, L115

Sigurdsson, S. \& Phinney, E. S. 1995, ApJS, 99, 609

Warren, S. R., Sandquist, E. L., \& Bolte, M. 2006, ApJ, 648, 1026

Zaggia, S. R., Piotto, G., \& Capaccioli M. 1997, A\&GA, 327, 1004

Zinn, R. \& Searle, L. 1976, ApJ, 209, 734 